\title{
A gaze interactive assembly instruction with pupillometric recording
}

\author{
John Paulin Hansen ${ }^{1}$ (D) Diako Mardanbegi ${ }^{2}$. Florian Biermann ${ }^{3} \cdot$ Per Bækgaard ${ }^{4}$
}

Published online: 6 July 2018

(C) Psychonomic Society, Inc. 2018

\begin{abstract}
This paper presents a study of a gaze interactive digital assembly instruction that provides concurrent logging of pupil data in a realistic task setting. The instruction allows hands-free gaze dwells as a substitute for finger clicks, and supports image rotation as well as image zooming by head movements. A user study in two LEGO toy stores with 72 children showed it to be immediately usable by 64 of them. Data logging of view-times and pupil dilations was possible for 59 participants. On average, the children spent half of the time attending to the instruction (S.D. 10.9\%). The recorded pupil size showed a decrease throughout the building process, except when the child had to back-step: a regression was found to be followed by a pupil dilation. The main contribution of this study is to demonstrate gaze-tracking technology capable of supporting both robust interaction and concurrent, non-intrusive recording of gaze- and pupil data in-the-wild. Previous research has found pupil dilation to be associated with changes in task effort. However, other factors like fatigue, head motion, or ambient light may also have an impact. The final section summarizes our approach to this complexity of real-task pupil data collection and makes suggestions for how future applications may utilize pupil information.
\end{abstract}

Keywords Gaze interaction · Instructions · Usage log · Pupil dilation · Pupillometry · Effort · Engagement · Task analysis · User experience · User interfaces

\section{Introduction}

Gaze-tracking sensors are emerging in consumer-grade PCs and head-mounted displays. Gaze interaction is convenient, particularly when the hands are occupied with a primary task, for instance assembling or cooking (Mardanbegi, Hansen, \& Pederson, 2012); gaze may then be used for input while handling objects and without contaminating the display itself.

In addition to the advantages of being hands-free, gaze interaction provides new opportunities to collect real-time

John Paulin Hansen

jpha@dtu.dk

1 DTU Management Engineering, Technical University of Denmark, Diplomvej, Building 371, 2800

Kgs. Lyngby, Denmark

2 School of Computing and Communications, Lancaster University, Lancaster, LA1 4WA, UK

3 Computer Science Department, IT University of Copenhagen, 2300 Copenhagen, Denmark

4 DTU Compute, Technical University of Denmark, 2800 Kgs. Lyngby, Denmark user data. How frequently is each element on a display attended to, and which elements are ignored? Central to this paper, previous research on pupil dilation has revealed a relationship between pupil dilation and cognitive effort. Under laboratory conditions with restrictions on head motion and under well-controlled light conditions, several studies have shown that when mental load increases, the pupils dilate proportional to the increase (e.g., Beatty \& Lucero-Wagoner, 2000; Kahneman \& Beatty, 1966) until the task demands become limited by available resources (Granholm, Asarnow, Sarkin, \& Dykes, 1996). This provides a unique possibility to identify, e.g., particularly demanding parts of a task and to quantify changes throughout task engagement-for both individuals and for groups of users. However, it is an open question if pupil data of relevance may be recorded in real-life situations without control of ambient light and with free head movements.

This paper also addresses some of the challenges for a widespread exploitation of gaze interaction in ubiquitous displays: Firstly, gaze sensors should be able to provide interaction opportunities comparable to those replaced, for instance finger tap selections, swiping, and pinching on a touch screen. Secondly, since most people are not yet familiar with gaze applications, they should be 


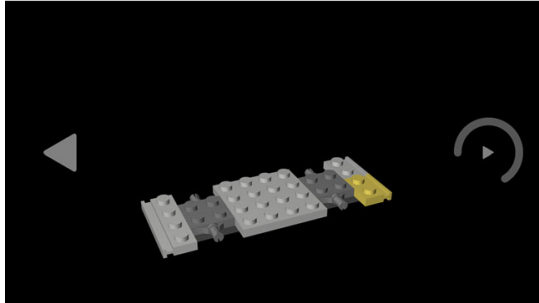

Fig. 1 The gaze interactive assembly instruction. Left: Stepping forward by dwelling at the arrowhead. A circle is drawn clockwise around the arrowhead to indicate how long it should be fixated. Right: The

self-explanatory with regard to their functional properties, for instance guiding users to position their heads within the track-box of the sensor. Finally, instant engagement should not be prevented by lengthy calibrations (Fig. 1).

We designed a digital LEGO construction manual to address some of these challenges and tested it in two toy stores. Forward and backward steps are made by gazing at arrow icons. The views of the LEGO model are controlled by head movements: leaning forward or backward makes the model zoom in or out; an interaction principle originally proposed by Harrison and Dey (2008). Moving the head sideways makes the model rotate left or right, cf. Fig. 2, bottom. Both eye and head movements are tracked by the very same gaze sensor (Mardanbegi et al., 2012).

The designers at LEGO were particularly interested in knowing more about the users' engagement during a building task. This information is very difficult for them to get without asking questions that would disturb the child. Measuring EEG, heart rate, or galvanic skin response requires wiring the children, which does not scale well to user studies with a high number of participants outside a lab. They would also like to know if some steps took an exceptionally long time since this might suggest to split them. Basic logging of user input provides the designers some insights, but won't tell if the time was spent on interpreting the pictorial instructions or working with the bricks. So, subsequently we analyzed the view times and pupil data recorded in the store to explore if changes would indicate also changes in performance during the building task.

Our paper has three contributions: We present an application where gaze provides a hands-free substitution for click, pitch, and swiping, and although not an entirely new approach, we suggest that it works well for assembly tasks like the one studied here and allows for easy behavioral data collection. We conduct a study to test if the instructions can be used immediately in a realistic task context. Finally, we analyze if the gaze data recorded may provide insights that could possibly be linked to how much attention sub-parts of the task received or to individual task events. Although focusing on a LEGO

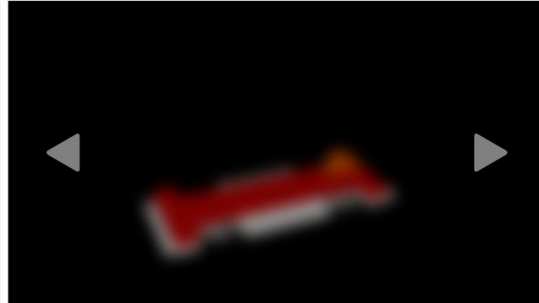

image gets blurred when the gaze tracker loses contact with the eyes, which provide immediate feedback to change the head position in front of the tracker

assembly task, we consider the application generic for a broad range of step-wise pictorial instructions typically found at workplaces, educational institutions, and in public domains. In laboratory work, for instance, a chemist is often required to follow specific procedures for handling substances. Gaze interaction with a workbench monitor would allow the chemist to navigate procedure descriptions without contaminating the display. At the same time, gaze tracking would be able to confirm that each instruction had been carefully studied. Similarly, students may engage with digital educational resources in a step-wise order, keeping both hands free for note taking. In this case, pupil data could indicate which parts of the learning material required the most mental effort from students.

\section{A gaze-interactive pictorial instruction}

Current digital versions of LEGO instructions for tablets ${ }^{1}$ present each next building step when pressing an arrowbutton in the right corner; going back is done by another button in the left corner. Zooming in on the model may be done by finger pitching while rotations may be done by swiping. We converted these basic functions to a gaze- and head-activated interface by having the stepforward/backward command done by dwelling on two arrow buttons shown on the right and the left sides of the screen, and the zooming and rotation done via head movement, s.c.f. Fig. 2, bottom).

The gaze-tracker model used for the experiment had a limited tracking box of approximately $\mathrm{x}=30 \mathrm{~cm}, \mathrm{y}=$ $20 \mathrm{~cm}$ and $\mathrm{z}=10 \mathrm{~cm}$ at $40 \mathrm{~cm}$ distance. Whenever gaze tracking was lost, which happened every time the child looked away from the screen, the image of the model would become slightly blurred. Only by looking at the screen and keeping the head within the tracking box would the child be able to regain a clear image. Preliminary tests with an early prototype had found this feedback to be effective; we observed that the children quickly learned how

\footnotetext{
${ }^{1}$ See for instance https://goo.gl/h4KUMF.
} 


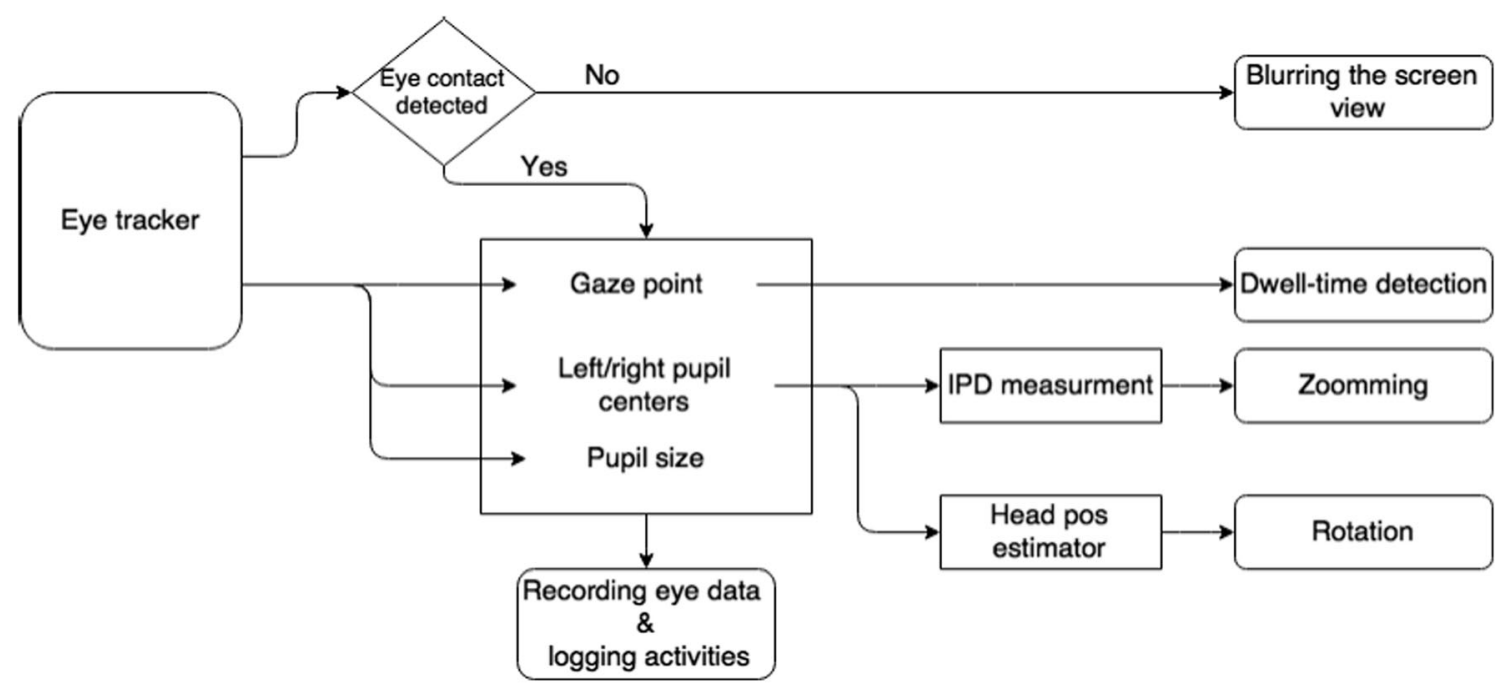

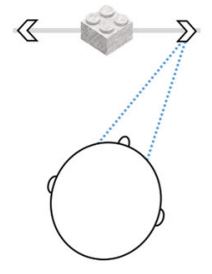

a

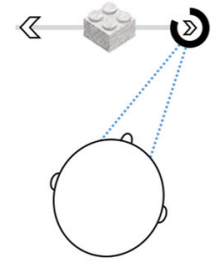

b

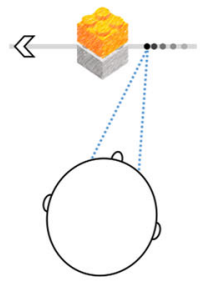

C

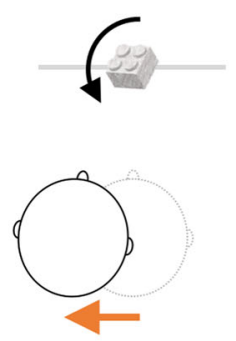

d

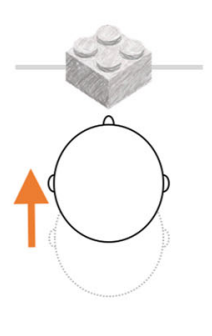

e

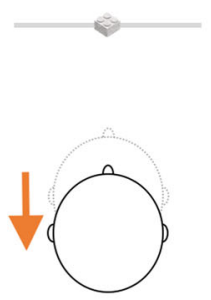

$f$

Fig. 2 Top: Flowchart of the gaze-interactive system showing how the information obtained from the eye tracker is mapped to different functions; IPD = inter-pupillary distance. The "head pos estimator" estimates the horizontal offset of the head vs. the center of the bounding box. Bottom: Interaction with the LEGO construction manual on a tablet using gaze and head movements. The user looks at the right

to reposition their head within the invisible boundaries of the sensors view angle whenever their head had been outside the box. In addition, the intro screen of the manual showed nothing but a LEGO head symbol and a small rectangle in the center. The head followed the movements of the child's head. The child was asked to place it within the small rectangle, which would then launch the instruction application, initially teaching the child where to position the head in front of the monitor.

The active areas of the dwell buttons are located on either side of the screen, covering the entire screen height and 20\% of the screen width, each. However, the visible size of the buttons does not cover the same area; the users see only an arrow in the middle of each activation areas (Fig. 1). By this design, users experience a good tracking precision without requiring high-precision tracking equipment and without a need to calibrate the gaze tracker for each user. Based on observations in a pilot study, we decided to set dwell-time to $600 \mathrm{~ms}$. Previous work on dwell-time gaze interaction technique has shown that dynamic feedback during the

arrow button to go to the next step (a), the button gets activated after dwelling (b), once dwell-time is completed, a moving circle guides the user's gaze back towards the digital model where new bricks are highlighted in yellow (c). Users can rotate the digital model by moving their head to the sides (d). Zooming can be done by leaning forward and backwards (e and f)

dwell-time activation can significantly improve the users' performance and reduce subjective workload (Majaranta, MacKenzie, Aula, \& Räihä, 2006). Thus, we provided two different kinds of feedback. During the dwell time, a visual feedback is given by shrinking the arrow and making it smaller while an animated circle is drawn around the arrow indicating the dwell time progression (Fig. 1; left). If the user looks away from the arrow before the dwell-time is finished, the dwell-time timer and all the animated feedback will reverse. This gives the system more robustness against potential noise or inaccuracy in the gaze tracking. Upon dwell-time completion, there will be a short click sound and then a small animated circle will move from the dwell button towards the center of the screen, guiding the user's gaze back towards the model again. New bricks added in each step gets highlighted in yellow.

The user can zoom in and out the view of the digital model by leaning forward and backwards towards the screen. Similar ideas has been tested before using a camera and face tracking, e.g., by Harrison and Dey (2008). 
However, we just use data from the gaze sensor to estimate the relative distance between the viewer and the screen; no extra cameras are implied (for more details on how to do this, see the "Apparatus" section).

\section{Related work}

Video-based analysis of manual activities and logging of input to digital devices are standard methods in usability studies. Recently, gaze tracking has become more common because it provides additional information on how much time each area is looked at, which elements that tends to get overlooked, and which parts are frequently revisited (Bojko, 2013). Measures and visualizations of gaze movements are widespread within experimental psychology (Holmqvist et al., 2011) and eye movement recordings have been used in numerous research projects on naturalistic tasks (Land \& Tatler, 2009).

Gaze interaction is now common practice within assistive technology (Majaranta et al., 2011), and a substantial amount of HCI research has explored the potentials of this modality, e.g., Sibert and Jacob (2000) and Zhai (2003). Standard input methods are dwell-time activation, (i.e., looking at a target for a set time, for instance $500 \mathrm{~ms}$, e.g., Ware \& Mikaelian, 1987); stroke activation (i.e., looking in one or several directions, in a consecutive order, with a saccade in between, e.g., Drewes \& Schmidt, 2007); and pursuit activation (i.e., following a smoothly moving target area, e.g., Vidal, Bulling, \& Gellersen, 2013). Current gazeinteraction research focuses on challenges and potentials in smart-phone interaction (e.g., Rozado, Moreno, Agustin, Rodriguez, \& Varona, 2015), smart-watches (e.g., Hansen et al., 2016), ubiquitous displays (e.g., Velloso, Wirth, Weichel, Esteves, \& Gellersen, 2016) and head-mounted displays (e.g., Itoh \& Klinker, 2014).

The pupil dilates when people increase effort. Measuring changes in effort during a task or differences in task loads between design alternatives may thus provide insights to developers of, e.g., learning tools and applications. Pupil data can be recorded with modern gaze-tracking technology used for gaze interaction. A majority of previous research on pupil dilations has been conducted with experimental or simulated tasks in a lab using either a chin rest or a headmounted eye-camera (cf. Klingner, Kumar, \& Hanrahan, 2008). There are good reasons for this limitation, as pupil changes caused by changes in scene brightness may equal or exceed those resulting from changes in effort. For instance, Ahern and Beatty (1979) reported task-evoked pupillary response (TEPR) dilations and the darkness response both to be on the order of $0.5 \mathrm{~mm}$, compared to a light reflex induced contraction of $1.5 \mathrm{~mm}$. The TEPR is consistent with previous works of Kahneman and Beatty (1966), and also the works of Klingner et al. (2008). Orlosky et al. (2017) reports load-induced pupil dilations of approximately $10 \%$.

Furthermore, it has been shown by Bradley, Miccoli, Escrig, and Lang that pupil size is modulated by activation of the (sympathetic) nervous system when viewing affective pictures (Bradley, Miccoli, Escrig, and Lang, 2008). There is a growing body of evidence that the pupil size is related to neural activity in the locus coeruleus (Eckstein, GuerraCarrillo, Singley, \& Bunge, 2017; Gilzenrat, Nieuwenhuis, Jepma, \& Cohen, 2010; Joshi, Li, Kalwani, \& Gold, 2016; Murphy, O'Connell, O'Sullivan, Robertson, \& Balsters, 2014), which is linked to arousal and attentional processes (Aston-Jones, Rajkowski, \& Cohen, 1999) and utility optimization and the dichotomy between exploration and exploitation (Aston-Jones \& Cohen, 2005). Thus, the pupil size is influenced by complex emotional and cognitive processes, not all of which we can control in the present experimental setup.

Finally, in visual tasks, the pupils are constantly moving with the eyes and the eye region moves with the head. So it is still an open question if tracking of pupil dilations is feasible outside a lab where changes in ambient light are unknown and where users are free to move their head as the task requires. To our knowledge, the present work is the first to combine gaze interaction with pupil data logging in a naturalistic task setting involving inexperienced users.

\section{User study}

Shops and shopping malls are attractive locations for user studies aiming at a high number of observations because they offer participants who can be recruited easily. In-store testing has a number of challenges, though: available space may be limited, lighting conditions may not be controlled, and participants may be distracted by noise and other shoppers coming by. We addressed these challenges by designing a simple interface, for an experiment that would last less than $20 \mathrm{~min}$, and by having adult assistants guarding each participants at all time.

\section{Participants}

A total of 72 participants were recruited among children accompanied by an adult in two LEGO stores, located in Germany and Denmark. All of the children were between 6 and 12 years, regular users of mobile phones and/or tablets, and all of them were familiar with LEGO model construction. None of them were wearing glasses or contact lenses. Consent was obtained from the accompanying adult to record data and video. 


\section{Apparatus}

We used two Microsoft Surface Pro tablets with Windows 8, which executed the instruction application, conducted gaze tracking, and logged use data. A 30-Hz low-cost (100 US\$) binocular gaze tracker from The Eye Tribe was used for the experiment. It recorded binocular gaze data when the participants were looking at the tablet, through a USB 3.0 with a claimed accuracy of 0.5 to 1.0 degree of visual angle for the gaze point. Additionally, a linear pupil size estimate is returned by the tracker in arbitrary units, with no specified accuracy. The algorithm behind this estimate is proprietary, but publicly proposed (Lin, Pan, Wei, \& Yu, 2010) methods involve fitting an elliptical shape to the outline of the pupil, correcting for the angle of view and returning the estimated radius or diameter of the pupil, which would allow the estimate to obtain subpixel resolution. This is also the method suggested by Klingner et al. (2008). We have no reason to believe the Eye Tribe tracker will do worse than publicly available algorithms. At viewing distances between 10 and $40 \mathrm{~cm}$, a typical pupil size that varies between 2 and $5 \mathrm{~mm}$ will have a viewing angle between 0.29 and 2.9 degree seen from the tracker. Assuming a native resolution of $1 \mathrm{~min}$ of viewing angle ${ }^{2}$, this corresponds to a range between 18 and 175 pixels, with a typical $3.5 \mathrm{~mm}$ pupil at $35 \mathrm{~cm}$ distance recording at approximately 34 pixels.

The Eye Tribe tracker has been compared against other trackers with respect to gaze points (Dalmaijer, 2014; Ooms, Dupont, Lapon, \& Popelka, 2015)and pupil size estimates (Dalmaijer, 2014), and has previously been used to assess load (Čegovnik, Stojmenova, Jakus, \& Sodnik, 2018). For the later, no quantifiable accuracy was derived, but it was qualitatively demonstrated to produce similar results to an EyeLink 1000 in controlled conditions, concluding that "the spatial precision and accuracy are good enough for fixation checking, point-of-regard analyses, and pupillometry" (Dalmaijer, 2014) for the Eye Tribe tracker.

The assembly model was a LEGO Mini VW T1 Camper Van (Model \# 40079), featuring 76 pieces and targeted at children 6 years and above. The original paper manual for this model had divided the instruction into 18 pictures. We

\footnotetext{
${ }^{2}$ In an experiment performed by the authors, the IR camera used by the tracker reports a maximum resolution of $2304 \times 1136$ pixels at $27 \mathrm{~Hz}$ when queried through the USB port using SmartGaze (Hume, 2016). The bounding box inside which the pupils are correctly detected at a distance of approximately $36 \mathrm{~cm}$ from the tracker is $25 \mathrm{~cm}$ wide, which is equivalent to a viewing angle of 38 degrees seen from the tracker. This would indicate that the native resolution of the camera corresponds to an approximate angle of view of 0.017 degrees (or 1.0 arcminute) or equivalently 60 pixels/degree.
}

could reproduce them digitally by use of a 3D model and in addition the model would allow users to zoom and rotate every image. The original paper manual consists of 18 steps with three of the steps broken further down into sub-steps. Our digital version showed all of the steps one-by-one, ending up with a total of 27 views. For zooming, we use inter-pupillary distance (IPD) as a heuristic to approximate the distance between a user's head and the screen. The IPD is calculated as the Euclidean distance between the estimated center positions of the pupil, which are returned by the eye tracking as coordinate sets $(x, y) \in[0 . .1]^{2}$ inside an imagined bounding box. We only zoom if the absolute difference of the current to the last stable IPD is equal to or larger than $\epsilon=0.01$ and some zoom factor $D=11$. The function $f_{\text {zoom }}(d)=(d \cdot D)^{2}$ gives the distance from the 3D model to the camera for a stable IPD $d$. The last IPD for which zooming occurred is now regarded as the last stable IPD. Using a last stable IPD prevents jitter. We linearly interpolate between camera distances over $200 \mathrm{~ms}$. Zooming is absolute.

We use the relative position of the user's head vs. the center of the screen to initiate a continuous relative rotation of the 3D model. ${ }^{3}$ Head coordinates are computed as the center point between both pupil centers. If the user's head is more than $1 / 3$ left or right of the center of the tracking bounding box, a continuous rotation of the 3D model in the opposite direction starts. We use the distance of the head to the closest point on the bounding box to determine rotation speed, where a larger distance results in a faster rotation. The rotation speed is given in degrees by $f_{\delta}(x)=$ $\operatorname{sgn}(x) \cdot x^{2} \cdot S$, where $x$ is the distance between the $x$ value of the head coordinates and the $x$ value of the closest point on the bounding box; $s g n$ is the sign function; and $S$ some configurable rotation speed factor, in our setup $S=10^{-5}$. The value of $f_{\delta}$ is added to the current camera angle at screen-refresh rate. The bounding box was positioned at tracking image center with a width of 300 pixels.

\section{Procedure}

The child was seated comfortably at a table in front of one of two tablets, which was positioned approximately $40 \mathrm{~cm}$ away and $20 \mathrm{~cm}$ above the table by use of a bendable tablet holder; see Fig. 3. The session started by asking the child to position a LEGO figure head on the screen inside a box by moving his or her own head. This would start a short 1-min interactive training session where the child would forward and reverse a three-step instruction and try out zooming and rotation. After this introduction, the study was started by launching the full instruction and pulling the 76 LEGO pieces out on the table. The children conducted the

\footnotetext{
${ }^{3}$ Rotating or tilting the head itself is not meant to initiate any action.
} 


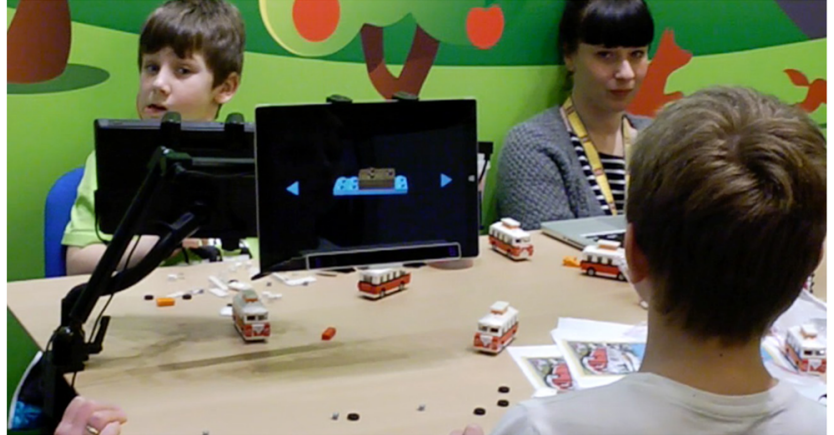

Fig. 3 Two children using the gaze-interactive building instruction in a toy store with an adult assistant next to them

building process by themselves and only got adult assistance if they were unable to continue on their own. After the experiment, all participants were given a small LEGO box as appreciation for their participation. In total, the session lasted between 15 and $20 \mathrm{~min}$.

\section{Design and analysis}

The study was performed as an observational study with all of the participants having one single exposure to the same condition.

During each session, all data from the eye tracker as well as all user-initiated navigation and zooming actions were recorded for subsequent analysis. From this recorded data, we derived the following measures for our study: task time for each building step (i.e., the cumulative time displaying instructions belonging to each step); view time per step (i.e., the cumulative time where gaze positions are recorded, indicating eyes on the tablet, within that step), play time per step (i.e., task time subtracted view time), number of regressions (i.e., how many times did the child go back to a previously visited step) and what fraction of the view time was spent during regressions vs. the total view time, for each step. Zoom and rotations were also counted.

Eight participants were removed from the analysis because they did not complete the building process. This was due to problems with gaze tracking, mostly because the children did not understand to re-position their head correctly in front of the tracker. Of the remaining 64, five had an uneven distribution of recorded pupil data with more than $75 \%$ in either the first or second half of the session, and were also removed from the analysis.

The pupil size estimate from the eye tracker varies between the values 17.0 and 67.1 (the lowest 5\% percentile and the highest $95 \%$ percentile across all participants) with an overall median value of 32.5. With an assumed pixelresolution of 1 arcminute, the median value corresponds to a pupil diameter of $3.3 \mathrm{~mm}$ at a distance of $35 \mathrm{~cm}$. At the same distance, the lowest value corresponds to $1.7 \mathrm{~mm}$ and the highest to $6.8 \mathrm{~mm}$ (although at a distance of $10 \mathrm{~cm}$ the highest value would correspond to only $2.0 \mathrm{~mm}$ ).

Before use, the pupil size estimates from the participants were filtered as follows: First, samples where data are only available for one eye are discarded. Then, in an effort to remove outliers, a Hampel filter (with a radius of 13 samples at $30 \mathrm{~Hz}$, and nsigma of 3) was applied, and in the process any outliers that have up to 13 neighbors within the window are replaced; this also interpolates blink periods up to $400 \mathrm{~ms}$ with the median value of a rolling window over the surrounding pupil size estimates. The linear pupil size estimate is hereafter compensated for changes in the calculated IPD, and any samples where the IPD is less than $50 \%$ of the median value over the session are further removed. The average Pearson correlation coefficient $R$ between left and right eye is hereafter 0.83 ; this indicates that the variations of the (independently estimated) left/right eye pupil sizes are strongly correlated, and rather than being artifacts of the measurement may point towards a common causal factor. Finally, the pupil size estimates are converted to a relative baseline, the median value across the experiment for each participant. ${ }^{4}$ In the absence of an absolute, linear (metric) pupil size estimate, this is done in order to calculate aggregate values in a comparable way across participants, resulting in a metric similar to the "percent in change pupil size" originally proposed by Hess (1972). Further, when comparing pupil size estimates between (sets of) tasks, we use an average PCPS (APCPS) across the periods where the participant is engaged in the selected tasks, similar to Iqbal, Zheng, and Bailey (2004), Iqbal, Adamczyk, Zheng, and Bailey (2005), and Bailey and Iqbal (2008).

\section{Results}

A large majority, namely 64 of the 72 participants (i.e., $88 \%$ ), managed to complete the model, and we were able to get a full gaze data set from 59 participants. On average, the 59 children spent $14 \mathrm{~min}$ and $20 \mathrm{~s}$ (S.D. $6 \mathrm{~min}$ and $33 \mathrm{~s}$ ) on the task. They made 5.6 regressions (S.D. = 5.6) and conducted 176 zoom- and 67 rotation- actions in average (S.D. 161 and 44 respectively), all indicative of the differences between individual children. It is, however, not possible to tell if the zoom and rotations were done on purpose or just happened as an effect of the child moving. Hence, we do not investigate any correlation between zoom and rotations and other factors.

\footnotetext{
${ }^{4}$ Note that we avoid using a $\mathrm{z}$-score normalization, as we cannot, and need not, make any assumptions as to whether the individual pupil size variations are equal across all participants.
} 


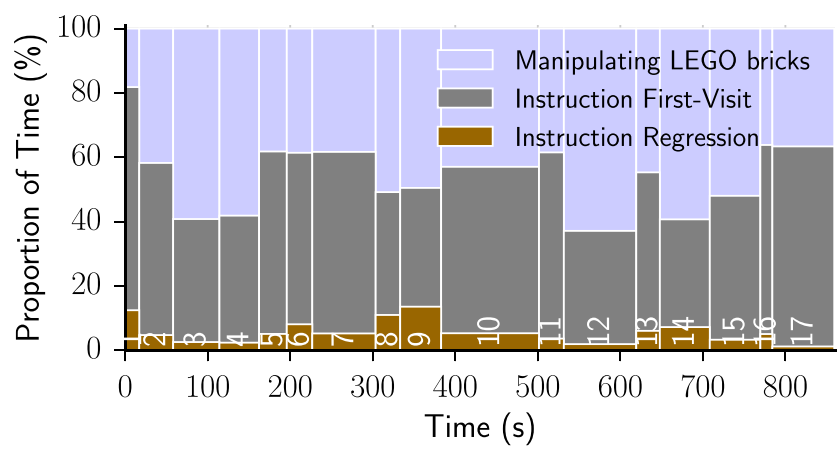

Fig. 4 Average time spent within each building step ( $x$-axis) with the stacked bars showing the proportion of the time spent either manipulating bricks (light blue) or when the gaze point was at the tablet showing the Instruction Manual ( $y$-axis); the later measure is divided into first-visits (gray) and regressions (yellow). In most steps, between 40 and $60 \%$ of the time is spent studying the instruction manual, with an average of $49.4 \%$

\section{View times}

The width of each column in Fig. 4 shows the mean duration of each individual step. The step division corresponds to the one used in the paper manual; in our digital version, there were two views for step seven, three for step 10, and four for step 17. The bars shows the distribution of time within each step: The light blue bar shows the proportion of time spent manipulating LEGO bricks ("play time"), the gray bars show the time spent looking at the instructions in the first visit, and the colored bars show the time spent in a regression: a subsequent visit to the step once a later step had commenced. The total time spent looking at instructions, the "view time", was on average $49.4 \%$ (S.D.: $10.9 \%$ ), i.e., roughly half of the time was spent studying the instruction manual. However, some of the children spent a lot of time looking at the tablet while assembling the model; the most extreme case would look at it $78 \%$ of the time. Others looked at the tablet more rarely; one of the participants only viewed it $27 \%$ of the time.

Figure 5 shows the progress of the building process relative to the accumulated time: instruction view time (green dashed line) and brick play time (red solid line). Note that the progress is remarkably linear, for both, indicating a steady pace through the construction. The Pearson correlation between view and play time was $R=$ $-0.837, p<0.001$. Our analysis does not, however, intend to address the correlation between the design and the time spent executing the building process any further.

\section{Pupil dilations}

Figure 6 shows the average percent change in pupil size (APCPS) relative to the baseline, at each step, across all

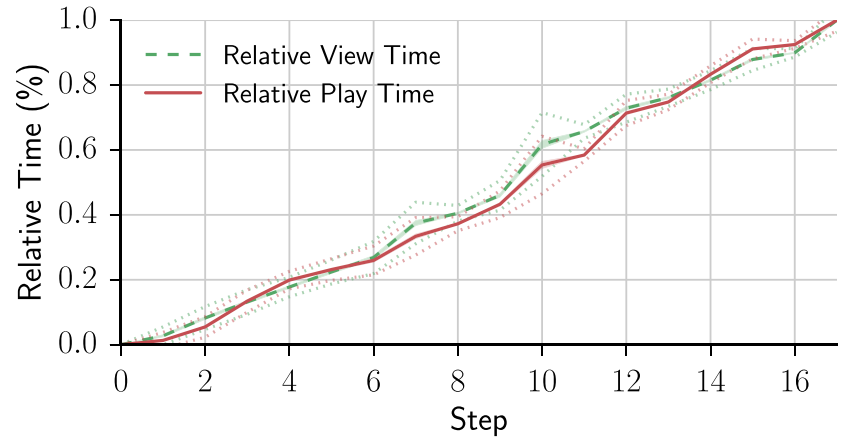

Fig. 5 Progress (in relative time) to completion of each step, shown as the view time (green dashed line) and playing time (red solid line). The shaded area behind each line indicates the standard error of the mean, and the dotted lines indicate the sample standard deviation

participants. The baseline for each participant is the overall median value of the pupil size estimate. The yellow graph depicts the uncorrected pupil size with the standard error of the mean shaded. The blue graph shows the same data after attempting to compensate for luminance: This was done by calculating the average screen luminance for each step from the image being shown (in relative units) as an approximation of the displayed luminance, and then regressing the pupil size against this approximated luminance, using a conventional linear regression model. The residual variance can then be taken as a representation of the luminance compensated pupil size, building on the premise that the pupil size can be modeled as the addition of two components, one caused by luminance and the other by other (subjective) factors, as demonstrated by Pfleging, Fekety, Schmidt, and Kun (2016). This does not account for the response time of the pupillary light reflex (Beatty \& Lucero-Wagoner, 2000), and is thus only a coarse approximation. Both graphs show an initial increased

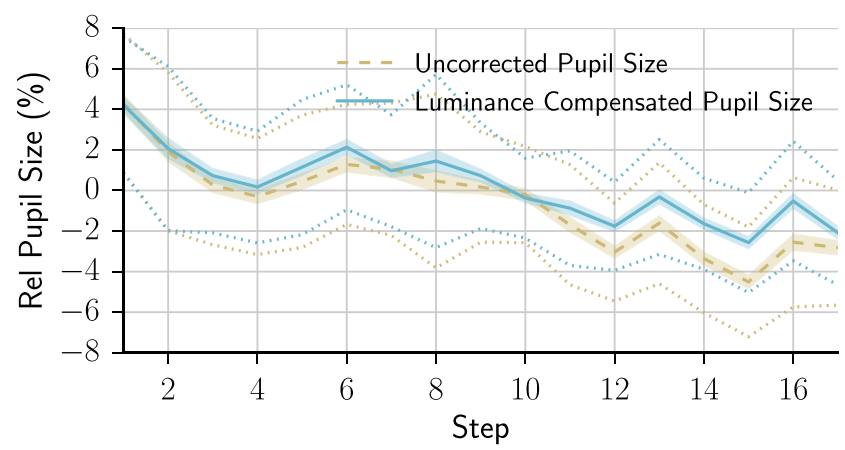

Fig. 6 Mean pupil size across all participants relative to their individual baseline, at each building step (uncorrected (yellow dashed line) and after luminance correction (blue). The shaded area behind each line indicates the standard error of the mean, and the dotted lines indicate the sample standard deviation 
pupil size and an overall declining trend. The effect of the luminance is mainly visible towards the end, where an increasing number of white bricks in the model made the screen brighter; however both graphs have similar declining trends. Note that as the eye tracker is only able to record data when the participant is looking directly at the screen and with the head inside the bounding box, no data is available when the participant looks at the bricks on the table; in our terminology, we only have pupil data for the view time and not for the play time.

Following the analysis method by Hyönä, Tommola, and Alaja (1995), we divided the tasks into three phases: beginning (steps 1 to 6), middle (steps 7 to 12), and end (steps 13 to 17), in order to compare the task-internal variation in pupil size. The mean APCPS values were 1.74, 0.02 , and $-1.43 \%$ with a standard error of the mean of $0.27,0.20$, and 0.18 , respectively. There was a significant effect, as evidenced by a non-parametric Kruskal-Wallis test $H=77.7$ and $p<0.000001$, and a subsequent post hoc analysis yielded pairwise Wilcoxon comparisons at the $p<0.00001$ values between any of the three divisions.

Inspired by the work of Bailey and Iqbal (2008), we looked further into the changes in pupil size when people advanced and regressed between steps. Figure 7 shows the difference in APCPS calculated as the average value over the time spent in first visit vs. the average value over the time spent in any subsequent regressions to the same step. On average pr. step, the participants spent $25.0 \mathrm{~s}$ viewing the instructions, and all valid gaze data, grouped accordingly into first and subsequent visits for each step, has been included in the APCPS metrics. Apart from the initial phase comprised of steps 1 to 3, there is a difference of APCPS when comparing first visit to regressions (subsequent visits), at the confidence levels indicated in Fig. 7 ( $p<0.05$ in blue and $p<0.01$ in green). With the exception of steps $6,12,15$, and 17 , the difference is statistically significant and is approximately $3 \%$.

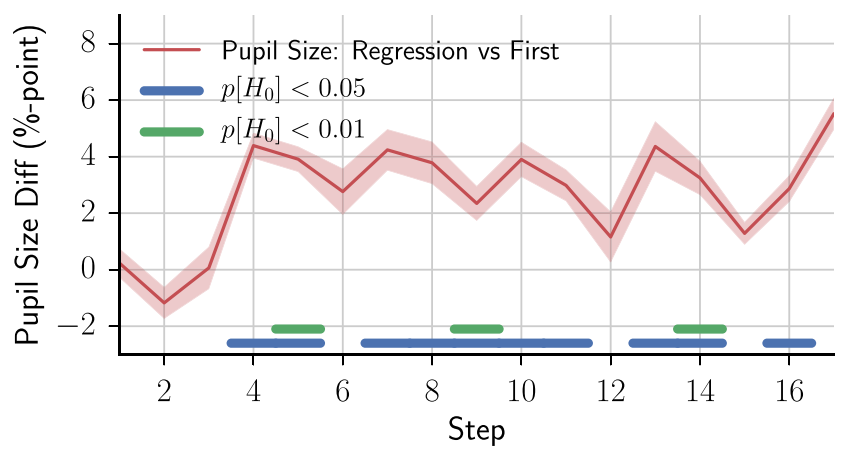

Fig. 7 Pupil size difference between first visit and regressions at each step $(r e d)$, with standard error of the mean indicated as a shade. Confidence levels that the values are different (at 5 and 1\%) also indicated (blue and green, respectively)

\section{Discussion}

The concept of gaze-interactive instructions, which additionally allows for collecting gaze and pupil data for subsequent analysis, was effective for the children, as evidenced by their successful use of the navigational features. The two main questions raised by LEGO designers, namely if there are indications of changes in engagement and if some steps stands out as more difficult, can be answered partially.

Measures of average time per task step and time spent looking at instructions (view time) were easy to collect and are not confounded by luminance variations or head movements. In our case, these metrics showed signs of a well-designed instruction manual with no individual steps standing out. The gains of collecting view time in addition to a standard log of button activations were, however, not obvious for this model, since view time and step time correlated well.

By tracking pupil size and compensating for head movements, we were able to document (A) an overall decreasing trend throughout the building task, indicated by a significant drop in the APCPS, and (B) a significant difference of most regressions compared to first visit to each step. With the generally accepted link between changes in pupil size and changes in effort, our results may suggest that (1) a higher effort is required initially (what Hyona refers to as the familiarity effect (Hyönä et al., 1995)), and (2) regressions, indicative of task problems, require additional effort, implied by significant increases in pupil size for regressions made at 11 out of 17 steps. Note that we have not used the trend observed in (A) to detrend (Strauch, Georgi, Huckauf, \& Ehlers, 2015) the APCPS before performing step (B), but have chosen to report the two results independently of each other; if we had done so the differences would have been (slightly) larger, since regressions by definition appear later than first visits.

However, it cannot, on the basis of this study alone, be concluded that the changes are caused solely by differences in effort; factors like increasing fatigue, changes in motivation or engagement, frustration, or other emotional factors could also possibly impact the results. From this perspective, the pupil size differences between regressions and first visits to a building step are more intriguing, and may more strongly suggest that cognitive or emotional factors as the underlying cause. It is, however, not possible to say whether the regressions made the pupil dilate because they made the children frustrated or the children exerted themselves - maybe even both. With a broad analogy, pupil changes may be like a gauge showing how fast a vehicle is running, but we do not know whether it's because the vehicle is going downhill or because the accelerator is activated. In any case, knowing that a specific type of 
event (i.e., a regression) consistently correlate with changes in the observable pupil size suggest it is relevant to keep track of that particular type of event. Further research could potentially identify other types of events, such as prearranged surprises or rewards, that may have similar effects on pupil dilation. Eventually, applications could dynamically monitor the flow of such events that have shown a correlation to pupil size changes, avoiding, for instance, giving rewards just after a surprise, or suggesting to take a break after a period with unusually high pupil dilations. It is an open research question if this micromanagement of events on the basis of pupil size measures would create a better overall user experience or improve efficiency.

A decrease in arousal caused by fatigue, or getting used to the task and the environment leading to habituation, may also play a role behind the observed decrease in pupil size throughout the building session, even though the children did not show other signs of being tired out, and were eager to finish the session, even those who could had difficulties using the application. Although we cannot at present point to the key causal factors, our analysis nevertheless showed a significant difference between the start, the middle, and the final part, while Hyönä et al. (1995) only found a significant decrease between the first and the middle division, not the last two. This suggests future studies to investigate if changes in dilation are different for different tasks across the full task and between task divisions, and additional experimental methods to identify and isolate the independent factors affecting the pupil size estimates.

Measuring pupil dilations in-the-wild is especially warned against because the impact of luminance changes are considered larger than the effects of task related changes. In the current study, we made the recordings in shop areas with homogeneous ambient light. Our observations suggest that in some situations similar to ours, where the luminance of the objects being viewed (here: the tablet display) can easily be determined, they could also to some degree be compensated for by standard regression methods.

We were not able to estimate or correct for all types of head pose changes based on the inter-pupillary distance alone, which could therefore be an additional confounding factor. ${ }^{5}$ Hence, it is advisable to use eye trackers for future

\footnotetext{
${ }^{5}$ For instance, rotating the head would also show as a reduced interpupillary distance, however it would also make one pupil appear slightly large and the other slight smaller, which would not be entirely consistent with the high positive Pearson correlation coefficient $R$ found between left and right eye pupil sizes
}

studies that have robust head-movement/pose estimation (Al-Rahayfeh \& Faezipour, 2013) for applications such as the one suggested here.

Future research should investigate if view time, regressions and pupil measures would correlate with other biometric measures, such as blink rate, heart rate variability, EEG, or galvanic skin response. If so, this would be a strong argument for including pupil dilation metrics as first choice because they are non-invasive, continuous, and have low latency (Bailey \& Iqbal, 2008).

Pupil metrics are particularly prospective if on-line gaze interactive building instructions are provided for augmented reality glasses, since the metrics could then be collected in continuous large-scale studies, and changes in illumination compensated for by analyzing the image data from the build-in image sensor of the augmented reality glasses. In the future, pupil metrics may even be used to ensure every builder being rightly challenging by offering an instruction dynamically adapting to the level of effort shown by the individual builder.

We used a well-designed instruction manual on a small-scale model that has been on the market for many years. However, the results seem to suggest that the concept could also be used for, e.g., early testing of new models, for $\mathrm{A} / \mathrm{B}$ testing different versions, or verifying against specific segments (age, gender, nationality, previous building experience, etc.). It may also be of some use comparing metrics between different models. Finally, by introducing steps that are deliberately designed to be difficult, for instance with many bricks added at once, the impact on effort, view time and regressions could be further examined.

\section{Conclusions}

Using a low-cost $30-\mathrm{Hz}$ gaze tracker in an unconstrained, real-task setting, we have provided successful gaze and head interaction for a large majority of the participants trying it. Concurrently, view time and pupil dilation metrics can be recorded, and we suggest this could potentially provide pupil data reflecting changes in cognitive or emotional states and effort, both across the task and evoked by regressions between tasks steps, when confounding factors to some degree can be accounted for or averaged out over many participants.

Acknowledgements The work was in part supported by Innovation Fund Denmark through the project Eye Tracking for Mobile Devices. Thomas Hoger, 3spin GmbH, conducted part of the user study. Lego A/S provided building material and feedback. The Eye Tribe helped developing the application. 


\section{References}

Ahern, S., \& Beatty, J. (1979). Pupillary responses during information processing vary with scholastic aptitude test scores. Science, 205(4412), 1289-1292. https://doi.org/10.1126/science.472746

Al-Rahayfeh, A., \& Faezipour, M. (2013). Eye tracking and head movement detection: a state-of-art survey. IEEE Journal of Translational Engineering in Health and Medicine, 1, 2100212. https://doi.org/10.1109/JTEHM.2013.2289879

Aston-Jones, G., \& Cohen, J. D. (2005). An integrative theory of locus coeruleus-norepinephrine function: adaptive gain and optimal performance. Annual Review of Neuroscience, 28, 403450. https://doi.org/10.1146/annurev.neuro.28.061604.135709

Aston-Jones, G., Rajkowski, J., \& Cohen, J. (1999). Role of locus coeruleus in attention and behavioral flexibility. Biological Psychiatry, 46(9), 1309-1320. https://doi.org/10.1016/S0006-3223 (99)00140-7

Bailey, B. P., \& Iqbal, S. T. (2008). Understanding changes in mental workload during execution of goal-directed tasks and its application for interruption management. ACM Transactions on Computer-Human Interaction, 14(4), 21:1-21:28. https://doi.org/10.1145/1314683.1314689

Beatty, J., \& Lucero-Wagoner, B. (2000). The pupillary system. In Handbook of psychophysiology, (Vol. 2 pp. 142-162).

Bojko, A. (2013). Eye tracking the user experience. Brooklyn: Rosenfeld Media.

Bradley, M. M., Miccoli, L., Escrig, M. A., \& Lang, P. J. (2008). The pupil as a measure of emotional arousal and autonomic activation. Psychophysiology, 45(4), 602-607. https://doi.org/10.1111 /j.1469-8986.2008.00654.x

Čegovnik, T., Stojmenova, K., Jakus, G., \& Sodnik, J. (2018). An analysis of the suitability of a low-cost eye tracker for assessing the cognitive load of drivers. Applied Ergonomics, 68, 1-11. https://doi.org/10.1016/j.apergo.2017.10.011

Dalmaijer, E. (2014). Is the low-cost Eye Tribe eye tracker any good for research? (Tech. Rep.) PeerJ PrePrints. https://doi.org/10.7287 /peerj.preprints. $585 \mathrm{v} 1$

Drewes, H., \& Schmidt, A. (2007). Interacting with the computer using gaze gestures. In Proceedings of the 11th IFIP TC 13 international conference on human-computer interaction - volume part ii (pp. 475-488). Berlin: Springer.

Eckstein, M. K., Guerra-Carrillo, B., Singley, A. T. M., \& Bunge, S. A. (2017). Beyond eye gaze: What else can eyetracking reveal about cognition and cognitive development? Developmental Cognitive Neuroscience, 25, 69-91. https://doi.org/10.1016/j.dcn. 2016.11.001

Gilzenrat, M. S., Nieuwenhuis, S., Jepma, M., \& Cohen, J. D. (2010). Pupil diameter tracks changes in control state predicted by the adaptive gain theory of locus coeruleus function. Cognitive, Affective, \& Behavioral Neuroscience, 10(2), 252-269. https://doi.org/10.3758/CABN.10.2.252

Granholm, E., Asarnow, R. F., Sarkin, A. J., \& Dykes, K. L. (1996). Pupillary responses index cognitive resource limitations. Psychophysiology, 33(4), 457-461. https://doi.org/10.1111/ j.1469-8986.1996.tb01071.x

Hansen, J. P., Lund, H., Biermann, F., Møllenbach, E., Sztuk, S., \& Agustin, J. S. (2016). Wrist-worn pervasive gaze interaction. In Proceedings of the ninth biennial ACM symposium on eye tracking research \& applications (pp. 57-64). New York: ACM. https://doi.org/10.1145/2857491.2857514

Harrison, C., \& Dey, A. K. (2008). Lean and zoom: Proximityaware user interface and content magnification. In Proceedings of the SIGCHI conference on human factors in computing systems (pp. 507-510). New York: ACM. https://doi.org/10.1145/ 1357054.1357135
Hess, E. H. (1972). Pupillometrics: A method of studying mental, emotional and sensory processes. In Greenfield, N. S., \& Sternbach, R. A. (Eds.) Handbook of psychophysiology (pp. 491531). New York: Holt, Rinehart \& Winston.

Holmqvist, K., Nyström, M., Andersson, R., Dewhurst, R., Jarodzka, H., \& Van de Weijer, J. (2011). Eye tracking: A comprehensive guide to methods and measures. London: Oxford University Press.

Hume, T. (2016). WIP Open-source IR eye tracker for the Eye Tribe tracker hardware [trishume/SmartGaze]. GitHub repository. Retrieved from https://github.com/trishume/SmartGaze

Hyönä, J., Tommola, J., \& Alaja, A. M. (1995). Pupil dilation as a measure of processing load in simultaneous interpretation and other language tasks. The Quarterly Journal of Experimental Psychology, 48(3), 598-612. https://doi.org/10.1080/14 640749508401407

Iqbal, S. T., Adamczyk, P. D., Zheng, X. S., \& Bailey, B. P. (2005). Towards an index of opportunity: Understanding changes in mental workload during task execution. In Proceedings of the SIGCHI conference on human factors in computing systems (pp. 311-320). New York: ACM. https://doi.org/10.1145/1054972.1055016

Iqbal, S. T., Zheng, X. S., \& Bailey, B. P. (2004). Task-evoked pupillary response to mental workload in human-computer interaction. In CHI '04 extended abstracts on human factors in computing systems (pp. 1477-1480). New York: ACM. https://doi.org/10.1145 1985921.986094

Itoh, Y., \& Klinker, G. (2014). Interaction-free calibration for optical see-through head-mounted displays based on $3 \mathrm{~d}$ eye localization. In 2014 IEEE symposium on 3d user interfaces (3dui) (pp. 75-82).

Joshi, S., Li, Y., Kalwani, R., \& Gold, J. (2016). Relationships between pupil diameter and neuronal activity in the locus coeruleus, colliculi, and cingulate cortex. Neuron, 89(1), 221-234. https://doi.org/10.1016/j.neuron.2015.11.028

Kahneman, D., \& Beatty, J. (1966). Pupil diameter and load on memory. Science, 154(3756), 1583-1585. https://doi.org/10.1126 /science.154.3756.1583

Klingner, J., Kumar, R., \& Hanrahan, P. (2008). Measuring the taskevoked pupillary response with a remote eye tracker. In Proceedings of the 2008 symposium on eye tracking research \& applications (pp. 69-72). New York: ACM. https://doi.org/10.1145 /1344471.1344489

Land, M., \& Tatler, B. (2009). Looking and acting: vision and eye movements in natural behaviour. London: Oxford University Press.

Lin, L., Pan, L., Wei, L., \& Yu, L. (2010). A robust and accurate detection of pupil images. In 2010 3rd international conference on biomedical engineering and informatics, (Vol. 1 pp. 70-74). https://doi.org/10.1109/BMEI.2010.5639646

Majaranta, P., Aoki, H., Donegan, M., Hansen, D. W., Hansen, J. P., Hyrskykari, A., \& Räihä, K.-J. (2011). Gaze interaction and applications of eye tracking: Advances in assistive technologies. Hershey: Information Science Reference - Imprint of: IGI Publishing.

Majaranta, P., MacKenzie, I. S., Aula, A., \& Räihä, K.-J. (2006). Effects of feedback and dwell time on eye typing speed and accuracy. Universal Access in the Information Society, 5(2), 199208. https://doi.org/10.1007/s10209-006-0034-z

Mardanbegi, D., Hansen, D. W., \& Pederson, T. (2012). Eye-based head gestures. In Proceedings of the symposium on eye tracking research and applications (pp. 139-146). New York: ACM. https://doi.org/10.1145/2168556.2168578

Murphy, P. R., O’Connell, R. G., O'Sullivan, M., Robertson, I. H., \& Balsters, J. H. (2014). Pupil diameter covaries with bold activity in human locus coeruleus. Human Brain Mapping, 35(8), 41404154. https://doi.org/10.1002/hbm.22466

Ooms, K., Dupont, L., Lapon, L., \& Popelka, S. (2015). Accuracy and precision of fixation locations recorded with the low-cost eye tribe 
tracker in different experimental setups. Journal of Eye Movement Research, 8(1). https://doi.org/10.16910/jemr.8.1.5

Orlosky, J., Itoh, Y., Ranchet, M., Kiyokawa, K., Morgan, J., \& Devos, H. (2017). Emulation of physician tasks in eye-tracked virtual reality for remote diagnosis of neurodegenerative disease. IEEE Transactions on Visualization and Computer Graphics, 23(4), 1302-1311. https://doi.org/10.1109/TVCG.2017.2657018

Pfleging, B., Fekety, D. K., Schmidt, A., \& Kun, A. L. (2016). A model relating pupil diameter to mental workload and lighting conditions. In Proceedings of the 2016 CHI conference on human factors in computing systems (pp. 5776-5788). New York: ACM. https://doi.org/10.1145/2858036.2858117

Rozado, D., Moreno, T., Agustin, J. S., Rodriguez, F. B., \& Varona, P. (2015). Controlling a smartphone using gaze gestures as the input mechanism. Human-Computer Interaction, 30(1), 34-63. https://doi.org/10.1080/07370024.2013.870385

Sibert, L. E., \& Jacob, R. J. K. (2000). Evaluation of eye gaze interaction. In Proceedings of the SIGCHI conference on human factors in computing systems (pp. 281-288). New York: ACM. https://doi.org/10.1145/332040.332445
Strauch, C., Georgi, J., Huckauf, A., \& Ehlers, J. (2015). Slow trends. In Proceedings of the 2nd international conference on physiological computing systems (pp. 61-66).

Velloso, E., Wirth, M., Weichel, C., Esteves, A., \& Gellersen, H. (2016). Ambigaze: Direct control of ambient devices by gaze. In Proceedings of the 2016 ACM conference on designing interactive systems (pp. 812-817). New York: ACM. https://doi.org/10.1145 12901790.2901867

Vidal, M., Bulling, A., \& Gellersen, H. (2013). Pursuits: Spontaneous interaction with displays based on smooth pursuit eye movement and moving targets. In Proceedings of the 2013 ACM international joint conference on pervasive and ubiquitous computing (pp. 439448). New York: ACM. https://doi.org/10.1145/2493432.2493477

Ware, C., \& Mikaelian, H. H. (1987). An evaluation of an eye tracker as a device for computer input2. In Proceedings of the SIGCHI/GI conference on human factors in computing systems and graphics interface (pp. 183-188). New York: ACM. https://doi.org/10.1145 129933.275627

Zhai, S. (2003). What's in the eyes for attentive input. Communications of the ACM, 46(3), 34-39. https://doi.org/10.1145/636772.636795 